\title{
Eduvisionando, edumisionando Chile 2017-2057: algunos desafíos experimentales, políticos y ejecutivos
}

\author{
Dr. Raúl Pizarro ${ }^{1}$
}

RESUMEN

Este ensayo corresponde a síntesis de investigaciones experimentales Escuela + Familia que han producido aprendizajes de alta calidad en Castellano, Matemática e Inteligencias Múltiples, para alumnos de básica/primaria y enseñanza media/secundaria en Chile, Colombia y Nicaragua. Y para establecer desafíos educativos (2017-2057) con foco preferente en alumnos pequeños (0-I0 años) e intervenciones aula que involucren metodologías pedagógicas, interaccionales y evaluativas para nuevos límites de aprendizajes, mayor autonomía y soberanía intelectual.

Palabras clave: Síntesis experimentales Escuela + Familia, límites de aprendizajes, autonomía y soberanía intelectuales.

\section{Visions and Missions for formal Education, Chile 2017-2057: Some Experimental, Political and Executive Challenges} \begin{abstract}
Strategic Educational Planning and Implementation for Chile 2017-2057: Some Experimental, Political and Executive Challenges: this essay implies educational synthesis research School + Family for pursuing sig-nificant enhanced learnings in Castilian, Mathematics, Multiple Intelligence for primary and secondary students in Chile, Colombia and Nicaragua. It also pre-sents some educational challenges (2017-2057) especially for kids (0-I0 years old), dealing with instructional, interactional and evaluative methodologies for new limits of learning, greater authonomy and intellectual sovereignty.
\end{abstract}

Keywords: Experimental synthesis school + family, limits of learning, authono-my and intellectual sovereignty.

I Measurement, Evaluation and Statistical Analysis, University of Chicago. Académico Titular Evaluación, Universidad Playa Ancha, Chile. E-mail: rjepizarrosan@gmail.com 


\section{Introducción}

Presentamos en este ensayo nuestros estudios y experiencias experimentales educativas con niños y jóvenes de Colombia, Nicaragua y Chile, y algunos desafíos futuros que, pensamos, debieran acompañarnos por 40 años (2017-2057) por una educación de alta calidad y excelencia de aprendizajes (APs), que ayuden a generar condiciones, posibilidades, expectativas, contextos, proyectos esenciales de vida y productos que permitan aumentar la calidad de vida en Chile. Lo que exponemos aquí corresponde a logros reales con niños escolares. No representa aspiraciones ni expectativas desmedidas e imposibles. No son "pura poesía" o "eso es imposible", como hemos escuchado muchas veces de alumnos, padres y apoderados, profesores, académicos: ¿qué tiene que ver la pobreza económica y cultural con los APs de la escuela? Con la educación tradicional, casi todo. Con innovaciones validadas internacional y nacionalmente (automaticidad en lectura + curriculum del hogar mejorado en la ciudad de Quilpué, Chile, por ejemplo), poco o casi nada. Corresponden a datos reales como hacer leer en tres meses a los niños de escuelas públicas chilenas vulnerables de primero básico. Todos nuestros niños deben aprender a leer bien en primero y no en $4^{\circ}$ básico. O cómo desarrollar, aumentando significativamente las inteligencias múltiples y los APs de estudiantes de $2^{\circ}$ medio, en la Región Metropolitana.

Nuestras ideas y experiencias están contenidas en dos (2) títulos que implican algunos modelos teóricos, conceptualizaciones y focos experimentales. Ellos implican una visión y misión positivas de la educación, tendientes a potenciar elevados APs para casi todos los estudiantes. Y, por otro lado, algunos desafíos políticos educativos contenidos en el título, relacionado con proyecciones actuales y futuras para Chile, dedicado a una mezcla posible de cómo pensamos y operamos algunas políticas públicas y privadas en educación, como agente movilizador cultural, educacional, social y económico.

\section{Modelos teóricos, conceptualizaciones, focos}

Exponemos algunos desafíos políticos y ejecutivos educativos que se han pensado, difundido y aplicado internacional y nacionalmente, 
que se pueden extrapolar en parte como micro/macro investigaciones para Chile 2017-2057. Corresponden a nuestras experiencias al haber usado y contrastado (junto a académicos, estudiantes de pre y posgrado y profesores del sistema escolar) lenguajes descriptivos, experimentales, metodológico-métricos, cualitativos y meta-analíticos. Preferentemente a favor de innovaciones experimentales con niños y jóvenes $\left(\mathrm{I}^{\circ}-8^{\circ}\right.$.) de escuelas públicas y privadas de Colombia (métodos Escuela Nueva + Curriculum del Hogar Mejorado, Municipios de Funza y Madrid, Proyecto Erradicación del Trabajo Infantil (ETI), US Department of Labor y World Vision International-Colombia), Nicaragua (Programa educativo freiriano de alfabetización y acceso laboral PAEBANIC del Ministerio de Educación) y Chile (métodos Curriculum del Hogar Mejorado + Mastery Learning; Curriculum del Hogar Mejorado + Automaticidad en Lectura, Municipios de Quilpué, Viña del Mar y V Región; MIDAS-Counseling + MITATeaching interviniendo Inteligencias Múltiples y Educación en la Región Metropolitana: Proyectos FONDECYT N ${ }^{\text {os }}$ I930223, I960137, I04025I; Proyecto FONDEF No. D00II047; Proyectos Crisol Dorado y Umbral; Proyecto FONIDE N ${ }^{\circ} 22 \mathrm{I}$; Convenio de Desempeño CD/PMI UPA I203).

Aunque trabajamos casi tradicionalmente en la universidad, cuando se trata de intervenir a niños pequeños/futuros ciudadanos que compartimos, optamos por el diseño, la aplicación, el análisis y la evaluación de proyectos experimentales transversales o longitudinales. Así, hemos obtenido ganancias y deltas substantivos y significativos en dominios altamente simbólicos, como Lenguaje y Matemática (Conn, 20I7; Peltier \& Vannest, 20I7), y sintetizando preferentemente la escuela con la familia (Kirk, 1993; Heckman, 2012-2013; Epstein, I995, 2013; UPA, I203, 2016).

A pesar de su poca difusión en nuestras facultades de educación en Chile, concordamos con Campbell \& Stanley (1974, p. I I) cuando se declaran "partidarios del método experimental como único medio de zanjar las disputas relativas a la práctica educacional, única forma de verificar adelantos en el campo pedagógico y único método para acumular un saber al cual pueda introducírsele mejoras sin correr el riesgo de que se descarten caprichosamente los conocimientos 
ya adquiridos a cambio de novedades de inferior calidad" (Bloom, 1972, 1984; What Works, I984; Marzano, 2003; Glass, 2016).

Hemos oscilado entre micro investigación (APs notables en 4-8 años) y macro investigación (APs notables esperables en 8-40 años). Ello contradice a la educación clásica grupal que, al masificar cobertura, logra curvas normales de fuerte asimetría positiva: menor calidad y mayor inequidad (sociología educacional; efectos Mateo y Regresivo). No aceptamos que casi únicamente la calidad esté anclada al NSEC, ni que las escuelas traduzcan la sociedad (Bloom, I964, I972, I976, I985, I988; Coleman, I990; Edmonds, I979, 1986; Oakes, I990; Witte \& Walsh, I990; Kellaghan et al., I993; Csikszentmihalyi et al.,I993; Heckman, 2012-2013; Pizarro, 2008, 2012, 2017). Al respecto, cabe recordar a Shulman (I990, cf. Gess-Newsome, 20I3) cuando presenta sus contenidos profesionales, de disciplina y curricular (PCK) para impactar aprendizajes, oscilando desde paradigmas proceso-producto hasta ecología del aula.

Tenemos una perspectiva positiva de la educación. Pensamos educativamente la educación. Así, concordamos con Bloom (I968, 1976, 1985), Eisner (I991, 2002), Gardner (I994, 2016), Gardner et al. (200I) y sus modelos educativos-antropológicos-intelectualesartísticos-evaluativos que priorizan la capacidad humana para aprender a nivel de excelencia educativa, y que sostienen que provistos los contextos, medios y materiales más apropiados de aprendizajes (APs), casi todos (mayor que $80 \%$ ) pueden alcanzar elevados logros académicos (igual o mayor $80 \%$ correcto).

Hemos diagnosticado e intervenido a alumnos, escuelas y familias en áreas, tareas, objetivos, competencias de menores logros académicos (Pizarro, I99I; Pizarro et al., 2001, 2004, 2016), y nunca considerando a la escuela sola, sino en sociedad, con cuatro agencias educativas: familia, comunidad, grupo de pares, medios de comunicación social, cuya intersección tiende a provocar excelencia educativa para casi todos nuestros estudiantes (Bloom, I964; Janhom, 1984; Pizarro, I99I; Kellaghan et al.,I993; Epstein, I995, 2013; Gardner et al., 200I; Pizarro et al., 2008, 2016). Como sostuvimos más arriba, nuestras síntesis educativas experimentales 
han considerado a la sociedad escuela (educación formal) + familia (educación natural).

Nos parece deseable considerar conjuntamente la igualdad de oportunidades (ingreso) con la igualdad de metas (egreso), mediando entre ambas las diversidades de métodos y contextos (Bloom, I976, 1988; Eisner, I99I). Si proveemos a todos los estudiantes de lo mismo (profesor, sala, método, libro, test, tiempo), ellos terminan al revés de lo políticamente correcto (menor calidad y mayor inequidad educativas $=>$ efectos Mateo y Regresivo). Ergo, debiéramos diversificar accesos culturales, APs, metodologías, interacciones, evaluaciones. Al usar evaluación formativa (EF) y mastery learning (ML) podemos lograr deltas Glass + I sigma (P84 correcto para más del $80 \%$ alumnos), personalizando la instrucción grupal, usando correctivos entre EFs A y B, y amplificar entre 4-7 veces las metodologías en el mismo espacio, tiempo y con los mismos estudiantes y el mismo profesor (cf. Jornada Escolar Completa en Chile). Claro que EF y ML no son indicados para las disciplinas curriculares de Artes, Literatura, Ciencias Sociales. Ningún método es igualmente eficiente y eficaz para todos los profesores, contextos, alumnos, niveles educativos y disciplinas curriculares (Bloom, I968, 1978, 1987; Pizarro, I99I, 2008).

A su vez, los talentos (socialmente construidos) como las inteligencias múltiples (IMs: musical, espacial cinestésico-corporal, lingüística, lógico-matemática, interpersonal, intrapersonal, naturalista, fundadas en supuestos neurológicos, evolucionistas y transculturales) son desarrollables y menos estables que el CI (Bloom, I985; Gardner, I993, I994; Pizarro, I99I, 2006, 2009, 2010; Weber, 1999; Shearer, I999, 2007, 2008; Castillo et al., 2000; Gardner, Csikszentmihalyi \& Damon, 200I; Pizarro y Clark, 20I0): FONDEF D00II047, Universidad de Chile, 2000 en media: Castellano, Matemática, Ciencias, Historia), con delta $+0,38$ sigmas. Ahora, experimentaríamos con niños pequeños (cf. Zero \& Spectrum Projects, Harvard.edu): desafío para homogeneizar diferencias educativas por intervención de las 8 IMs (métodos MIDAS-Counseling más MITA-Teaching; Pizarro y Clark, 2010): 
By talent we mean an unusually high level of demonstrated ability, achievement, or skill in some special field of study or interest. (Bloom, I985, p. 5)

This is in contrast with earlier definitions, which equate talent with natural gifts and aptitudes. (Bloom, I985, p. 5)

The common attitude people hold toward "talent" or "giftedness" - such as a very high IQ or a prodigious musical ability - is that these traits are natural advantages with which some individuals had the good fortune to be born. But talent is not a natural category. Talent is a social construction: It is a label of approval we place on traits that have a positive value in the particular context in which we live. (Csikszentmihalyi et al., 1993, p. 23)

Una inteligencia es "la capacidad de resolver problemas, o de crear productos, que sean valiosos en uno o más ambientes culturales" (Gardner, I994, p. I0). Apoyamos a los teóricos mencionados precedentemente y en este mismo párrafo, en que el alto desarrollo intelectual, cultural, académico -world class students- no depende necesariamente ni del estrato, ni de los logros previos, ni de otras condiciones adscriptivas, sino de la calidad de los entornos/métodos donde participan nuestros alumnos en la escuela, familia, sociedad, grupo de pares, medios de comunicación social. Y de sus intereses, gustos, esfuerzos por estudiar/aprender a alto nivel (igual o mayor $80 \%$ correcto) (Bronffen-brenner, I979; Becker, I98I; Bloom, I984; Walberg, I984; Epstein, I995, 2013; What Works, I986; Shulman, I990; Marzano, 2003; Pizarro, 1991, 2008, 2009; Pizarro y Clark, 2007; Berns, 2007; Erhnam, 2009; Pizarro et al., 2016).

$\mathrm{Y}$, que provistas las mejores y más apropiadas condiciones previas y actuales de APs, lo que un estudiante puede lograr a un alto nivel, casi todos lo pueden aprender. Ello genera altos autoconceptos, autoestimas, APs (almacenados en la memoria larga, los cuales pueden ser accesados y vueltos a la realidad con poca práctica. Usted puede haber dejado de andar en bicicleta o patines por muchos años, pero basta un poco de ejercicio y recupera sus niveles previos: novicio $=>$ experto ... experto). También, automaticidades que liberan tiempo 
y conciencia: una persona puede ejecutar más rápido y pensando en varias cosas a la vez. Ellas construyen a su vez excelencia educativa, meritocracia, autonomía, creatividad y soberanía intelectual $=>$ límits of lear-ning, endstates (Bloom, 1968, 1972, 1978, I985; Husen, 1988, Husen \& Tuijnman, I991; Gardner, I994; Sternberg \& Grigorenko, 2001; Zhang \& Sternberg, 2006).

En términos contextuales y de desarrollo de mentes, nos parece que Eisner (I99I, 2002) acierta completamente al sostener que los niños nacen con cerebro, pero no con mente. Las escuelas son lugares para desarrollar mentes. Nuestros actuales/futuros estudiantes, ciudadanos cultos estudian 23-30 años y por ocho horas diarias $=\mathrm{I} / 3$ de su vida y la $\mathrm{I} / 2$ de su vida despierta. Debemos ir mucho más allá que la Tortuga Falsa ("Alice in wonderland"): "We had the best of educations -in fact, we went to school every day-" $=>$ cobertura $=>$ altas calidades y excelencias educativas, logrando política, metodológica y simultáneamente lo mejor del progresismo (equidad, derechos) junto a lo mejor del capital humano (libertad, meritocracia): efecto Robin Hood, mayor calidad y equidad educativas, curvas de fuerte asimetría negativa, tipo jota (Carroll, 1933, p. 92; Pizarro, 2008).

También es posible y deseable generar una elitización de la masa; o una masificación de la élite intelectual, para usar lenguajes de los años 1960-1970. Sostener a nivel nacional que la calidad educativa está anclada casi únicamente al NSEC es de un simplismo que linda con no haber tenido acceso a información directa y "fugitiva" (Lavin, 1968; Coleman, I990; ENIN, I969-20I6; MINEDUC chileno, 2000-2009; Rawls, 2003; Pizarro, 2008, 2009). O, demuestra cierta "inocencia educativa" (Bloom, I972): ¿correlación = causalidad?, suponer que las decisiones educativas no tienen respaldo investigativo suficiente, y priorizar siete focos/variables de fuerte impacto sobre los APs). O, pensar "falacias Simpson" por uso de información sintética-analítica (cf. big data, data mining) o, por uso no apropiado de lenguajes investigativos, confundir una correlación/asociación bivariada con causalidad. Es cierto que el NSEC está asociado a la calidad educativa cognitiva; pero no es el único factor vinculante o principal (como la inteligencia, la aptitud, el rendimiento académico 
previo), ni menos que la cause. El pasto está verde cuando llegan las golondrinas; pero estamos en primavera. Los niños escriben mejor cuando tienen las manos más grandes; pero también porque son mayores sus edades y niveles educativos (cf. Huff, I993; Bloom, 1972; Pizarro, 2012).

Al predecir descriptivamente APs cognitivos, los factores más asociados son los intelectuales, con 50\% de impacto (inteligencia, aptitud, rendimiento académico previo. Pero no siempre lo más impactante es políticamente correcto). Los factores de personalidad con I6\% (interés, salud mental, carácter, motivación, autoconceptos, habilidades blandas); finalmente, los factores de NSEC (educación y ocupación familia, tenencias, ingresos, inversión cultural) con impactos del 10\% (cf. Lavin, I968; Heckman, 2012-2013). Tales estudios implican correlaciones $\mathrm{r}=+0,3$ a $\mathrm{r}=+0,85$ (comunalidades entre $9 \%$ y $72,3 \%$ ).

Experimentalmente, ahora, es el método o la innovación, la intervención significativa de APs lo que importa educativa y científicamente (si p entonces q). Cabe recordar que tan importante como el azar para escoger grupos equivalentes (al menos 2), como en la asignación del tratamiento (p), luego de estudiar y seguir a algún teórico hay que pilotear tests y el método (varianza sistemática). Si su metodología funciona, se puede aplicar a micronivel hasta replicarlo a macronivel. En ello pueden pasar parsimoniosamente 7-I0 años (innovación => reforma; cf. Tyler, 1989). Más coloquialmente, si Ud. corre solo en un estadio, no puede llegar segundo. Aquí las correlaciones ideales entre el set de las 3 variables identificadas en el párrafo anterior, se esperan cercanas $(+/-0,30)$ o igual a cero. Las varianzas entre grupos, en cambio, grandes y a favor del grupo experimental o intervenido (Campbell \& Stanley, 1974; Shulman, I990; Kirk, I995; Kerlinger \& Lee, 2002; Pizarro, 2008).

Para seleccionar métodos y/o usar evaluaciones formativas, sugerimos usar Catálogos de Productividad Educativa (investigación metaanalítica) con distintos métodos mundiales rankeados por impacto sobre APs formales medidos en sigmas de la curva normal (Bloom, I984, 1987, con 3.000 investigaciones; Walberg, I984; Hedges y Olkin, I985; Pizarro, I99I, 2009, 2010; Glass, 2016, con más de 
400 meta-análisis y 50.000 investigaciones). Y, para interpretaciones substantivas, usar Tabla de Cohen (1988). Recordemos que el mejor método del mundo es el más antiguo (tutoría, con impacto $+2,0$ sigmas sobre grupo tradicional). Nadie la puede ejecutar masivamente a nivel país; pero sí a nivel micro (aula). El mejor ejemplo: Aristóteles haciéndole clases a Alejandro Magno; pero con la tenue diferencia en nuestra contra, que ni los profesores somos Aristóteles, ni nuestros alumnos hijos de reyes.

\section{Políticas e inversiones educativas futuras}

Ahora, en términos de política e inversión educativas, esperamos que los aportes económicos para profesores, escuelas, universidades y sistema científico sean entre 6-8\% PIB con I\%-2,5\% para Ciencia y Tecnología. Así podemos otorgar valor agregado a nuestra cultura, creatividad, producción, y poder participar en/de un mundo desarrollado y globalizado. Con el actual 0,38\% del PIB otorgado a Ciencia y Tecnología en el presupuesto nacional 2018, resulta difícil habilitar proyectivamente las interdependencias entre universidades, capital humano avanzado -formado o en formación-, empresas, mundo productivo y de servicios, respuestas eficientes a las necesidades de la población chilena actual y futura.

También, y puesto que todo Chile está de acuerdo, debiéramos priorizar políticas, inversiones y reformas cognitivas, afectivas, valóricas, psicomotrices en nuestros párvulos y estudiantes del sistema educativo público y privado. Y para considerar y agregar excelencia educativa (en vez de calidad, que nos mantiene desfasados hace 33 años) para nuestros futuros/presentes ciudadanos, tendríamos que compartir y realizar (coexistiendo con la educación tradicional) algunos de los siguientes pensamientos y acciones:

Diagnosticar e intervenir niños pequeños entrega muchos frutos educativos: las edades 0-I0 años son más que prioritarias. Son muy importantes algunos indicadores educativos cualitativos, tales como la búsqueda, la autonomía, el sello personal, la fantasía, la amistad, el compartir, la felicidad, el sentido y la ética, la creatividad, la trascendencia, el trabajo lúdico/disciplinado y bien hecho, etc. 
(Bloom, 1976, I985, 1987; Bronfenbrenner, 1979; Eisner, I99I, 2002; Csikszentmihalyi et al., I993; Gardner, 1994, 2005, 2016; Gardner et al., 200I; Heckman, 2012-2013; Ben-Shahar, 2009). Cosas tan elementales y gratis, pero que tienen mucho sentido e impacto actual y futuro: llamar a los párvulos, niños y jóvenes por el nombre y no por el apellido, o por número de lista; que los profesores sonrían más que de vez en cuando; que ellos sientan que son tomados en cuenta; que pertenecen a ambientes y grupos gratos, y que los tratamos muy bien.

Resulta altamente sugerible y prudente intervenir siete dominios (Feldman, 1994): Lenguaje (vocabulario, lectura, comprensión lectora, escritura, idiomas, símbolos y códigos culturales), Matemática y Computación (topología, geometría, aritmética, símbolos, códigos), Ciencias (método científico, problemas, hipótesis, generalizaciones, culturas, contextos), Estudios Sociales y Economía (caída a terreno, humanismo y artes liberales, necesidades e inversiones, tendencias, culturas), Artes (musicales y plásticas, escénicas, ballet, sinfónicas, símbolos y códigos, representaciones), Educación Física, Deportes y Recreación; desarrollo intrapersonal, interpersonal, afectivo, emocional, religiones, trascendencias, sentidos y significados. Participar activamente de una cultura desarrollada implica no solo ubicar estructuras simbólicas, sino comprender y aplicar creativamente sus códigos/"pinturas": palabra, corchea, número, códigos computacionales, movimientos, percepciones, sentimientos del ser humano, artes, etc. (Eisner, I99I, 2002; Gardner, I994, I995, 2005, 2016; Bloom, I985; Csikszentmihalyi et al., I993; Gardner et al., 200I; Ben-Shahar, 2009). Si los niños aprenhenden tales códigos y APs a temprana edad (sintetizando al menos escuela + familia) tendríamos, seríamos arquitectos, reinventaríamos (Bloom, I985, I987; Eisner, I99I; Csikszentmihalyi et al., I993; Gardner, I994, 2005, 2016; Gardner et al., 2001) un Chile con mejor calidad de vida para todos a futuro.

En términos de cantidad de cursos en la escuela y tiempo instruccional, todo aconseja sintetizar cursos (4-6 en Enseñanza Básica y 5-8 en Enseñanza Media), al igual que los países desarrollados. Con profesores que tengan entre I2-I8 horas de contacto pizarra/ 
medios/contextos/virtualidad; ganen alrededor de US\$3.0005.000 mensuales; y que asistan a la escuela mayor cantidad de días al año (240 similar a Japón o 220 como Cuba). Nuestros colegios subvencionados y particulares pagados casi llegan a 200-2I0, al igual que Alemania. Bastantes escuelas, liceos públicos y algunas universidades en Chile, alrededor de 180-200 días. Está muy probado que, a mayor calidad académica-docente, contacto, ganas y estudio en la escuela, mayores serán los APs; y viceversa (cf. Edmonds, I989; Witte \& Walsh, I990; Oakes, I990; Stodolsky, I99I; Jesson, 2002; Chávez et al., 2007; Pizarro, 2009, 2010; Vera, 2016; Aliaga, 2017).

Respecto a nuestra educación terciaria (crisol prioritario de generación creativa y difusión de conocimientos y desarrollo cultural, científico y tecnológico), resulta aconsejable discutir y precisar: (a) la cobertura y condiciones de la gratuidad, reduciendo deltas entre montos para asignarla y valor real de las carreras; (b) la estructura, administración, gestión, poder, accountability; autonomía, financiamiento, sueldos y honorarios; (c) las tipologías de universidades, academias y escuelas de las Fuerzas Armadas, IPs y CFTs, y duración de la educación terciaria: entre 2-2,5 para técnicos superiores y 4-4,5 para títulos más licenciatura; (d) la productividad académica (docencia, extensión, investigación, evaluación, asesorías, consultorías, "invisible college", artículos y libros, seminarios nacionales e internacionales, creatividad, propiedades intelectuales, patentes, licencias y royalties) y acreditación de tales instituciones, sus focos prioritarios e integraciones con entidades propias y externas. También, (e) la calidad y categorías de sus académicos y el respaldo y satisfacción usuaria y social; (f) títulos y grados académicos que otorgan y bajo cuáles condiciones (visiones, formación, modelos, misiones, competencias, duración, exigencias, perfiles, planes de estudio, mallas curriculares, programas cursos, aulas); (g) sana competencia por desafíos, servicios y recursos entre entidades estatales y privadas; (h) impactos, estabilidad, cambio y proyecciones de la academia, inversión en capital humano avanzado (becas, profesores visitantes, pasantías, intercambios académicos, años sabáticos); (i) mayor apoyo financiero y substantivo para las universidades estatales regionales de Chile; (j) reinversión en la propia academia y estructura universitaria de las ganancias de los ejercicios académicos anuales. 
Y, en términos de actuales y futuros impactos contextuales y directos en las enseñanzas y APs de nuestros estudiantes, podemos mencionar algunos tópicos a considerar: (a) desarrollo de talentos educativos, inteligencias múltiples (musical, cinestésico-corporal, espacial, lingüística, lógico-matemática, interpersonal, intrapersonal, naturalista) y habilidades blandas (empatía, liderazgo, trabajo en equipo y comunicación, metacognición, intereses y vocaciones, creatividad, innovación, asertividad, resiliencia, autoconceptos, ética y disciplina para realizar un trabajo bien hecho, etc.): ¿complementos para ingreso a la educación terciaria?; (b) manejo eficiente de big data (correlaciones, data mining, clusters, falacias Simpson) para explorar, analizar, evaluar políticas públicas y uso político y proyectivo de la evaluación, aporte societal democrático y transparente de la información; (c) contribución de la inteligencia artificial y la robótica en educación, especialmente en los medios de comunicación, kits robóticos educativos, TICs, redes sociales, diversificación de enseñanzas y APs, ahorro de tiempo, cambio de algunos roles educativos, ampliación de "pinturas, códigos y APs" en aulas y fuera de la escuela, nacional e internacionalmente (aldea global y sistema de créditos internacional), desarrollando curricula flexibles y diversos; (d) operación más continua de teorías de la mente y APs tácitos, implícitos motivadores y reforzantes de APs formales de la escuela. Aquí ayuda bastante el computer: lenguajes, programación, colores, reacciones, secuencias, textos, series on screen; (e) consideración más exigible entre diversos agentes educativos (Escuela + Familia + Sociedad, Comunidad + Grupos de Pares + Medios de Comunicación/Redes Sociales), cuya intersección/síntesis/ sociedad pueda producir excelencia educativa para casi todos nuestros estudiantes. El desafío es ir más allá de los estudios descriptivos típicos, en los que todos enseñamos con fuerte anclaje adscriptivo para tales síntesis; es decir, atreverse con teorías y lenguajes experimentales.

\section{Referencias bibliográficas}

Aliaga, B. S. (2017). Enseñanza de la Educación Física Infantil y su incidencia en la Participación. Tesis Magister en Ciencias de la Educación Física, Deportes y Recreación, UPLACED.

Becker, S. G. (198I). A teatrise on the family. Cambridge: Harvard University Press. 
Ben-Shahar, T. (2009). Even bappier: A gratitude journal for daily joy and lasting fulfilment. New York: McGraw Hill Companies.

Berns, M. R. (2007). Child, family, school, community ( $7^{\text {th }}$ Ed.). Canada: Thomson Wadsworth.

Bloom, S. B. (1964). Stability and change in buman characteristics. New York: John Wiley and Sons.

Bloom, S. B. (1968). Learning for mastery. UCLA Evaluation Comment, l(2), I-I2 (ERIC Document N ED053419).

Bloom, S. B. (1972). Innocence in education. Evaluation Comments, 8, I-I4.

Bloom, S. B. (1976). Human characteristics and school learning. New York: McGrawHill.

Bloom, S. B. (1984). The 2-Sigma problem: The search for methods of group instruction as effective as one to one tutoring. Educational Researcher, 13, 4-I6.

Bloom, S. B. (1985). Developing talent in young people. New York: Ballantine.

Bloom, S. B. (1986). The hands and feet of genius. Educational Leadership, 43 (5), 70-77.

Bloom, S. B. (1987). A response to Slavin's mastery learning reconsidered. Review of Educational Research, 57, 507-508.

Bloom, S. B. (1988). Helping all children learning well in elementary school and beyond. Principal, 67, I2I-I17.

Böhm, W. y Schiefelbein, F. E. (2004). Repensar la educación. Bogotá, D.C.: Editorial Pontificia Universidad Javeriana.

Bronfenbrenner, U. (I979). The ecology of buman development. Cambridge: Harvard University Press.

Campbell, T. D. y Stanley, J. (1974). Diseños experimentales y cuasi-experimentales en la investigación social. Buenos Aires: Amorrortu.

Carroll, L. (1933). Alice in wonderland. New York: Holt, Rinehart and Winston, Inc.

Castillo, N. J., Pizarro, S. R., Saavedra, L. M., Redondo, R. J., Alarcón, Q. D. y Juliá, M. (2000). La aplicación del paradigma de las inteligencias múltiples en el mejoramiento de la calidad de la educación media y la orientación vocacional. Proyecto FONDEF No. DOOII047, Universidad de Chile, 2000.

Chávez, M. M., Flores, L. A., Guioguiadez, O. K., Jeldes, O. G. y Ruiz, R. I. (2007). Relación entre escuelas efectivas y rendimiento académico en las escuelas 
municipales pertenecientes a las comunas de Viña del Mary Valparaíso. Tesis Título de Profesor de Educación Diferencial, Mención en Deficiencia Mental y al Grado Académico de Licenciado en Educación, Universidad de Playa Ancha de Ciencias de la Educación.

Cohen, J. (1988). Statistical power analysis for the behavioral sciences. Hillsdale: Lawrence Earlbaum Associates.

Coleman, S. J. (1990). Equality and achievement in education. Colorado: Westview Press.

Conn, M. C. (2017). Identifying effective education interventions in SubSaharan Africa: A meta-analysis of impact evaluations. Review of Educational Research, 87 (5), 863-898.

Csikszentmihalyi, M, Rathunde, K. \& Whalen, S. (1993). Talented teenagers. The roots of success and failure. New York: Cambridge University Press.

Edmonds, R. R. (1979). Effective schools for the urban poor. Educational Leadership, 37 (I), I5-24.

Edmonds, R. R. (I986). Characteristics of effective schools. En, U. Neisser, Ed., The school achievement of minority children: New perspectives (pp. 93-I04). Hillsdale, N.J.: Lawrence Erlbaum Publishers.

Eisner, W. E. (I99I). What really counts in schools. Educational Leadership, 48 (5), I0-I7

Eisner, W. E. (2002). The kind of schools we need. Pbi Delta Kappan, 83 (8), 576-583.

Epstein, L. J. (1995). School/Family/Community partnerships. Caring for the children we share. Pbi Delta Kappan, 76 (8), 70I-712.

Epstein, L. J. (2013). Programas efectivos de involucramiento familiar en las escuelas: Estudios y prácticas. Santiago de Chile: Fundación CAP.

Escalante, J. (I989). On creating ganas: A conversation with Jaime Escalante. Educational Leadership, 46 (5), 46-47.

Gardner, H. (1993). Estructuras de la mente. La teoría de las inteligencias múltiples (2a. Ed.). México: Fondo de Cultura Económica.

Gardner, H. (1994). Educación artística y desarrollo bumano. Buenos Aires: Paidós.

Gardner, H. (1995). Reflections on multiple intelligences. Myths and messages. Pbi Delta Kappan, 77 (3), 200-209.

Gardner, H. (2000). Intelligence reframed: Multiple intelligences for the 21 th century. New York: Simon \& Schuster. 
Gardner, H. (2003). 20 years of multiple intelligences. Reflections and a blueprint for the future. Keynote Address presented at The $84^{\text {th }}$ Annual American Educational Research Association, April 21, 2003, Chicago, USA.

Gardner, H. (2005). Las cinco mentes del futuro. Barcelona: Ediciones Paidós Ibérica, S.A.

Gardner, H. (2006). Inteligencias múltiples y liderazgo en los negocios y la educación. Viña del Mar: Seminario auspiciado por la Universidad de Valparaíso, Casino Municipal de Viña del Mar.

Gardner, H. (2007). Fireside chat: Good work in a global context. $88^{\text {th }}$ Annual Meeting of The American Educational Research Association (AERA), April 9-I4, 2007, Chicago, USA.

Gardner, H., Csikszentmihalyi, M. \& Damon, W. (200I). Good work. When excellence and etbics meet. New York: Basic Books.

Gess-Newsome, J. (2013). Pedagogical content knowledge. In John Hattie \& Eric M. Anderman (Eds.), International guide to student achievement (pp. 257-259). New York: Routledge.

Glass, V. G., Mcgraw, B. \& Smith, L. M. (I98I). Meta-analysis in social research. Beverly Hilss: SAGE.

Hedges, V. L. \& Olkin, I. (I985). Statistical metbods for meta-analysis. New York: Academic Press.

Huff, D. (1993). How to lie with statistics. London: Nolton and Company Ltd.

Husen, T. (1988). Nuevo análisis de la sociedad del aprendizaje. Barcelona: Paidós.

Husen, T. \& Tuijnman, A. (I99I). The contribution of formal schooling to the increase in intellectual capital. Educational Researcher, 20 (7), 17-25.

Janhom, S. (I984). Educating parents to educate their children. Unpublished doctoral dissertation, University of Chicago.

Jesson, F. D. (2002). Value added and the benefits of the specialism. London: Technology Colleges Trust.

Kellaghan, T., Sloane, K., Alvarez, B. \& Bloom, S. B. (I993). The bome environment and school learning. San Francisco: Jossey Bass Publishers.

Kerlinger, N. F. y Lee B. H. (2002). Investigación del comportamiento. Métodos de investigación en ciencias sociales ( $4^{a}$ Ed.). México: McGraw-Hill.

Kirk, E. R. (1995). Experimental design: Procedures for the behavioral sciences ( $3^{\text {a }}$ Ed.). California: Brooks/Cole Publishing Company.

Lavin, D. (I968). The prediction of academic performance. California: Russell \& Sage. 
Marzano, J. R. (2003). What works in schools: Translating research into action. Baltimore: ASCD.

Marzano, J. R. (2004). Building background knowledge for academic achievement: Research on what works in schools. Baltimore: ASCD.

Ministerio de Educación (2003). Estadísticas de la Educación 2001. Santiago de Chile: División de Planificación y Presupuesto.

Ministerio de Educación (2005). Indicadores de la educación en Chile 2000-2004. Santiago de Chile: División de Planificación y Presupuesto.

Oakes, J. (1990). What educational indicators? The case of assessing the school context. Educational Evaluation and Policy Analysis, I I (2), I8I-I99.

Palardy, J. G. \& Rumberger, W. R. (2008). Teacher effectiveness in first grade: The importante of background qualifications, attitudes, and instructional practices for student learning. Educational Evaluation and Policy, 30 (2), II I-I40.

Peltier, C. \& Vannest, J. K. (2017). A meta-analysis of schema instruction the problema-solving performance of elementary school students. Review of Educational Research, 87 (5), 899-920.

Pizarro, S. R. (1983). Validez predictiva del sistema de admisión a pedagogías, Academia Superior de Ciencias Pedagógicas de Valparaíso, 1981. Tesis de Magíster en Ciencias de la Educación, mención Medición y Evaluación, Pontificia Universidad Católica de Chile.

Pizarro, S. R. (1990). Lo que funciona en educación. Revista de Orientación Educacional, Universidad de Playa Ancha de Ciencias de la Educación, 6- 7, 46-56.

Pizarro, S. R. (1983). Teoría del rendimiento académico. Diálogos Educacionales, 6, 30-39.

Pizarro, S. R. (199I). Quality of instruction, bome environment and cognitive acbievement. Unpublished doctoral dissertation, University of Chicago.

Pizarro, S. R. (1994). Educational quality, curriculum of the home and math achievement. Paper presented at The Sixth Annual International Roundtable on Families, Communities, School and Children's Learning, April 4, New Orleans, USA. Paper presented at The First European Roundtable on Families, Communities, Schools and Children's Learning, September 7, Faro, Portugal. Proyecto FONDECYT 1930223, UPLACED,1993.

Pizarro, S. R. (2000). Análisis y evaluación del sistema educativo: Cobertura, equidad, calidad, excelencia y actualización. Valparaíso: UPLACED, Facultad de Ciencias de la Educación. 
Pizarro, S. R. (2008). Índices de Riesgo Educativo (IREs). Ponencia en el Foro Departamental sobre Evaluación Educacional, Gobernación de Educación, Departamento de Cundinamarca, I4 de agosto, 2008, Teatro Antonio Nariño, Bogotá, Colombia.

Pizarro, S. R. (2008). Sistemas educativos formales y efectos Mateo, Regresivo y Robin Hood. Boletín de Investigación Educacional, Pontificia Universidad Católica de Chile, 23, 2, I3-38. Ponencia en la Comisión Organizadora de Encuentros Nacionales e Internacionales de Investigadores en Educación (ENIN), 5 de Septiembre, Santiago de Chile, 2008; y en el $8^{\circ}$ Encuentro Interregional y $2^{\circ}$ Internacional de Investigadores en Educación, Universidad de Los Lagos, Osorno, Chile, I5-I6 Enero 2009.

Pizarro, S. R. (2009). Análisis encuestas laborales, proyecto ETI, Visión Mundial Colombia. Bogotá: Informe Proyecto ETI, VMC.

Pizarro, S. R. y Clark, L. S. (2000). Inteligencia múltiple lógico-matemática y aprendizaje escolar. Revista de Psicología de la Universidad de Chile, IX (I), 75-89.

Pizarro, S. R., Clark, L. S. y Machuca, S. L. (200I). Inteligencias múltiples y aprendizajes escolares. Boletín de Investigación Educacional, Pontificia Universidad Católica de Chile, 16, 289-300.

Pizarro, S. R., Colarte, T. P., Machuca, S. L., Donoso, Z. F., Martínez, C. M. y Walter, C. I. (2002). Análisis psicométrico de las escalas de inteligencias múltiples MIDAS-Kids. Revista de Psicología de la Universidad de Chile, XI (2), III-I24.

Pizarro, S. R., Clark, L. S. y Colarte, T. P. (2003). Inteligencias múltiples, curriculum del bogar, intereses, autoestimas y logros académicos previos y actuales, alumnos de educación media científico bumanista, Quilpué, Valparaíso, Cbile, 2004-2007: Estudio longitudinal y comparativo. Proyecto FONDECYT No. I04025I, UPLACED, 2003.

Pizarro, S. R. y Clark, L. S. (2007). Static and dynamic influences of multiple intelligences, curriculum of the home, interests, self-esteems, previous learning factors on current learning. $88^{\text {th }}$. Annual Conference of The American Educational Research Association (AERA-SIG: Multiple intelligences: Theory and Practice), April 9-I4, 2007, Chicago, USA. Proyecto FONDECYT No. I04025I, UPLACED, 2003.

Pizarro, S. R. y Clark, L. S. (2008). Inteligencias múltiples, curriculum del hogar, intereses, autoestimas, aprendizajes previos y actuales: investigación comparativa y longitudinal. Boletín de Investigación Educacional, Pontificia Universidad Católica de Chile, 23 (I), I I-40. Ponencia presentada en el IV Encuentro ENIN Interregional, Universidad de Concepción, noviembre 2008. 
Proyecto FONDECYT No I04025I, Universidad de Playa Ancha de Ciencias de la Educación, 2004-2007.

Pizarro, S. R., Clark, L. S. y Muñoz, B. M. (2008). Índices de riesgos educativos. Proyecto FONDECYT 2009, CONICYT-FONDECYT, Universidad de Playa Ancha de Ciencias de la Educación (UPLACED), Junio de 2008.

Rawls, J. (2003). A theory of justice. Massachusetts: Harvard University Press.

Schiefelbein, F. E. (1988). Siete estrategias para elevar la calidad y eficiencia del sistema educacional. Boletín Proyecto Principal de Educación en América Latina y el Caribe, 16. Santiago de Chile: UNESCO/OREALC.

Schiefelbein, F. E. (I99I). En busca de la escuela del siglo XXI. Santiago de Chile: CPU-UNESCO/OREALC.

Shearer, C. B. (1999). The MIDAS. A professional manual. USA: Greyden Press.

Shearer, C. B. (1999). The MIDAS challenge! USA: Greyden Press.

Shearer, C. B. (2007). Investigating relationships among multiple intelligences, reading and math test scores. $88^{\text {th }}$ Annual Conference of The American Educational Research Association (AERA-SIG: Multiple Intelligences: Theory and Practice), April 9-I4, 2007, Chicago, USA.

Shearer, C. B. (2008). Creating extraordinary teachers. USA: Greyden Press.

Shulman, S. L. (1990). Paradigmas y programas de investigación en el estudio de la enseñanza: Una perspectiva contemporánea. En Mervin C. Wittrock (Ed.), La investigación de la enseñanza (pp. 9-9I). Barcelona: Paidós Educador.

Sternberg, J. R. \& Grigorenko, L. E. (200I). Learning disabilities, schools, and society. Pbi Delta Kappan, 83 (4), 335-338.

Sternberg, J. R. (2007). Who are the bright children? The cultural context of being and acting intelligent. Educational Researcher, 36 (3), I48-I55.

Stodolsky, S. S. (I99I). La importancia del contenido. Barcelona: Paidós.

Tyler, W. R. (1989). An interview with Ralph Tyler. In George F. Madaus \& Daniel Stufflebeam (Eds.). Educational evaluation: Classic works of Ralph W. Tyler (pp. 243-272). Boston: Kluwer Publishers.

U.S. Department OF Education (1986). What works: Research about teaching and learning. Washington, D.C.: U.S. Government Printing Office.

Vera, P. (2016). Factores asociados a la motivación docente, Corporación Municipal de Renca, Región Metropolitana, Chile, 2014. Tesis Doctorado en Política y Gestión Educativa, Universidad de Playa Ancha de Ciencias de la Educación. 
Visión Mundial Colombia. (2009). Informe técnico final del proyecto erradicación del trabajo infantil ejecutado por Visión Mundial Colombia, en convenio con el Departamento del Trabajo de los Estados Unidos USDoL. Bogotá: Visión Mundial Colombia, Proyecto ETI.

Walberg, J. H. (I984). Improving the productivity of America's schools. Educational Leadership, 4l, 19-26.

Weber, E. (1999). Enfoque para enseñar las inteligencias múltiples (EEIM) como modelo para la reforma de la educación media y superior. Quinto Seminario Internacional de Innovación Educativa, INACAP, agosto I999.

Witte, J. F. \& Walsh, D. J. (1990). A systematic test of the effective schools model. Educational Evaluation and Policy Analysis, 12 (2), I88-2I2.

Zhang, L. \& Sternberg, J. R. (2006). The nature of intellectual styles. New Jersey: Lawrence Erlbaum Associates. 